\title{
Investigation of Correlation between the Temperature on Air-Snow and Snow-Ice Interfaces and the Atmospheric Air Temperature
}

\author{
Vadim K. Goncharov ${ }^{1}$, Natalia Yu. Edush ${ }^{1}$, Ekaterina S. Zueva ${ }^{1}$, Natalia Yu. Klementeva ${ }^{2}$, Jianmin Qin ${ }^{3,4}$, Liqin \\ Cui $^{3}$, Li Zhang ${ }^{3}$, Xiao Deng ${ }^{3,4}$ and Peng Cheng ${ }^{3,4}$ \\ 1. State Marine Technical University of Saint-Petersburg, Russia \\ 2. Krylov State Research Centre, Saint-Petersburg, Russia \\ 3. College of Physics and Optoelectronic, Taiyuan University of Technology, Taiyuan, China \\ 4. Key Laboratory Advanced Transducers and Intelligent Control System, Ministry of Education, Taiyuan University of Technology, \\ Taiyuan, China
}

\begin{abstract}
Solution of the practical problems of the ice engineering requires the data about the strength of the ice cover that depends upon its temperature. In most cases, the snow lies on the ice cover and the ice temperature differs from the atmospheric air temperature. To reveal the correlation of the air temperature with temperature on interfaces air-snow and snow-ice, the known in the thermophysics solution of the problem of the heat transfer through the multilayer plate was applied. Derived solution connects the temperature of air and temperature on the snow-ice interface and satisfactory correlates with data of the field measurements of the temperature within snow layer and ice cover and ice thickness on the Heilongjiang (Amur) River. Results of investigation are recommended for the ice temperature evaluation in engineering practice.
\end{abstract}

Key words: Snow, ice, air, temperature, interface, ice thickness.

\section{Introduction}

The snow cover is commonplace on the ice cover of the freshwater basins: rivers and lakes. The heat conductivity of the snow is considerably less than the ice heat conductivity. Therefore, the snow layer is peculiar heat insulation and it performs substantial part in the process of the ice cover formation that is the increasing of the ice cover thickness [1-3].

The thermodynamic processes that take place on the interfaces air-snow, snow-ice and ice-water are analyzed in the investigations devoted to the creation of methods for the forecast of the ice cover thickness on river and lakes [4-7]. Various theoretical and

Corresponding author: Vadim K. Goncharov, doctor of eng., professor, research fields: bubbles and drops dynamics, ice mechanics, ice propulsion of ships, safety of winter navigation and environment effect of crude oil and natural gas transportation. empirical representations for these processes are developed and applied where the determinant factor is the atmospheric air temperature. It revealed the considerable discrepancies between the calculated and measured ice cover thicknesses under comparison. It is possible to link this situation with the inadequate account of the part of the snow layer on the ice surface, because its thermophysical properties depend on many factors [8]. It is reasonable to take into account the part of the snow layer by means of averaging of the field measurements data. Such approach was used in Ref. [1]. The special coefficient that depends on the snow layer thickness was introduced in the formula connecting the ice thickness with the accumulated sum of the negative average daily temperatures of the air.

For many practical problems of the ice engineering solving that connected firstly with the fresh ice strength, which depends on its temperature [9], it is 
required the knowledge of the temperature on the upper surface of the ice cover (defining the ice strength) and temperature of the snow surface for the measured or forecasted temperature of the atmospheric air [10]. To establish this interdependence, it is expedient to apply the solution for the temperature stationary distribution across the multilayer plate known in the thermophysics [11].

In this study, the mentioned above solution was applied for the two-layer medium: snow and ice, at which the atmosphere and water space adjoin. In the winter conditions, the heat flow from the water to the atmosphere takes place through snow-ice cover of the underlying water space. This flow includes two components: the flow due to the difference between the water temperature and the atmospheric air temperature and the flow of the ice crystallization heat on the interface water-ice. The difference of temperature of water and air determines the first component. It is possible to determine the second component from the Stephen condition [12] for the immovable water space.

For rivers, where the water mass flows, for the Siberian rivers determined interconnection of the ice cover thickness with the accumulated sum of the negative average daily temperatures of the air [1] was applied. This empirical dependence was transformed into the rate of the ice thickness increasing and then into the ice crystallization heat flow with using the developed before methodology [13]. As result, the relatively simple formulas that connect the air temperature with temperature of the snow surface and the temperature of the ice cover surface were derived.

Results of computations by the produced formulas were compared with results of the field measurements of the temperature and thickness of the ice cover on the Heilongjiang River. The correspondence of calculated and measured data is satisfactory quite one.

\section{Statement of Problem}

During time, when it is possible to consider the winter weather as sustained one, the daily variation of the atmospheric air temperature is not large comparatively (no more than $1{ }^{\circ} \mathrm{C} /$ hour) and the sun radiation has small intensity. Accordingly, the air temperature variation occurs slowly and connected with one the temperature of the snow cover and the ice layer vary slowly also and within the narrow range. Therefore, it is possible to accept the following assumption: for each specific temperature of the atmospheric air, the quasi-stationary distribution of temperature inside the snow and ice layers is realized at some moment. Temperature on the border ice-water remains constant one and equals the temperature of the water (ice) freezing. The depth of the water body is large enough in comparison with the ice cover thickness, the water temperature gradient is small one and the thermal heat flow from bottom is not taken into account.

The heat propagation within system fresh water-ice-snow-atmosphere is considered. It is supposed the temperature of all mediums is constant one on the horizontal planes. It is analyzed the one-dimensional problem: the origin of $\mathrm{Z}$-axis places on the interface snow-atmosphere. The heat conductivity carries out the heat transfer within the snow and ice layers. The thermal boundary layer exists on the surface of the snow in which the convective diffusion of the heat in the atmosphere occurs (Fig. 1 illustrates the stated problem).

It is accepted following designations: $h_{\text {ice }}$ - the ice cover thickness and $\lambda_{\text {ice }}$-its coefficient of thermal conductivity, $h_{s n}$-the snow layer thickness and $\lambda_{s n}$-its coefficient of thermal conductivity, $T_{s / a}$-temperature on the interface snow-atmosphere, $T_{s / a}$ - one on the interface snow-ice, $T_{a}$ - temperature of the atmospheric air. Temperature on the interface ice-water corresponds one of the fresh ice crystallization $T_{w}=0{ }^{\circ} \mathrm{C}$.

The specific flow of heat (on the unit of area) from the water body to the atmosphere through the system of two solid and one gas medium in accordance with 

Interfaces and the Atmospheric Air Temperature

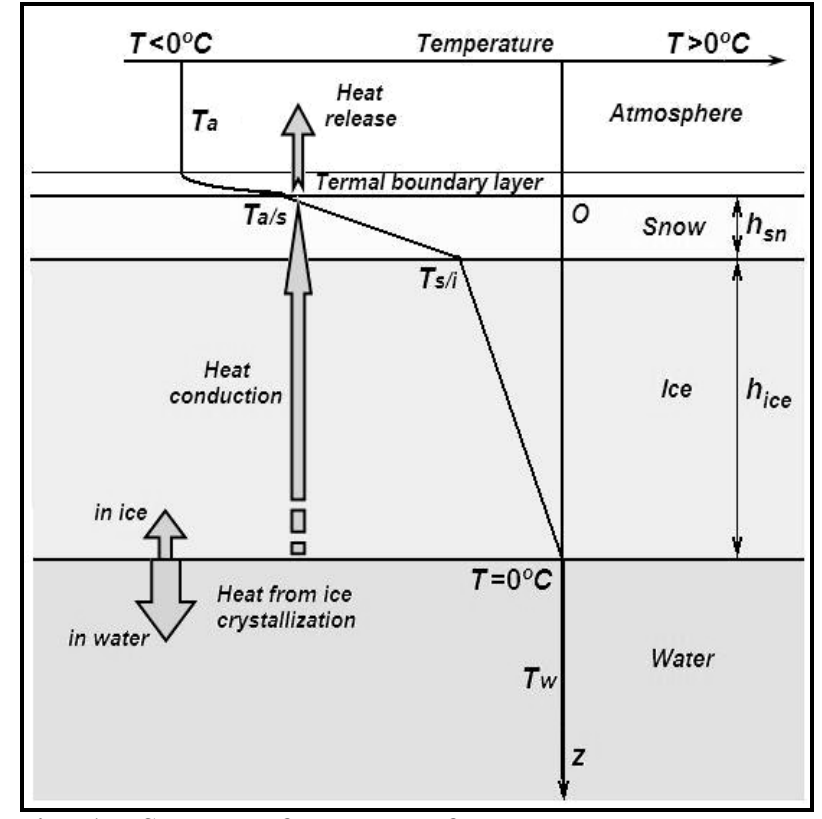

Fig. 1 Schema of the heat flow and the temperature variation in water, ice cover, snow layer and atmosphere.

Fourier law [2] can be presented as sum of two components. First, it is the heat flow from medium with higher temperature (water) to medium with lower temperature (atmosphere). Second, certain part $\mu$ of the heat flow from the ice crystallization on the interface water-ice (the rest part of heat diffuses in water body). The following form was derived (temperature of air $T_{a}<0$ always)

$$
q_{w a}=\frac{T_{w}-T_{a}}{R_{\text {sum }}}-\mu F_{h 0} T_{a} .
$$

In this formula $F_{h 0}$ - the heat flow from the ice crystallization, $\mu-$ coefficient that defines the part of this heat, which interpenetrates into ice cover (the other part of one-into the water body), $R_{\text {sum }}$ - coefficient of the thermal resistance (coefficient of the heat transfer) that connects with the thermal conductivities of the ice and snow and the convective heat release from the snow surface in the atmosphere $\left(\alpha_{s a}-\right.$ coefficient of the convective heat release)

$$
R_{\text {sum }}=\frac{1}{\alpha_{\text {sa }}}+\frac{h_{\text {ice }}}{\lambda_{\text {ice }}}+\frac{h_{\text {sn }}}{\lambda_{\text {sn }}} .
$$

We consider that the heat flow from ice crystallization is distributed between ice and water in proportion to their thermal conductivities. In this case the share of the crystallization heat that interpenetrates into the ice cover and then into the atmosphere should be presented as following:

$$
\mu=\frac{\lambda_{\text {ice }}}{\lambda_{w}+\lambda_{\text {ice }}} .
$$

For the specific flow of heat from the water body to the atmosphere determination, it is necessary first to parameterize the specific heat flow of the ice crystallization as function of the air temperature and the ice cover thickness. Because the results of analytical investigation are compared with the field measurements on the Heilongjiang River (the north-east China), the data obtained on the Siberian Rivers [1] were used to parameterize the heat flow from the ice crystallization.

\section{Heat Flow from the Ice Crystallization}

The intensity (rate) of the ice cover increasing depends on many factors, which characterize processes that occur in the atmosphere and on the medium interfaces [11]. It is difficult to predict these factors, so for the forecast of the ice cover thickness the empirical methods are applied, which use data of the multiyear observation of the air temperature and the ice cover thickness. These methods $[1,4,7]$ take into account and average the role of all processes that affect the ice cover freezing.

The following empirical formula approximates data of the field observations of the weather and the ice conditions on the Siberian Rivers [5] (for the snow layer thickness under $0.2 \mathrm{~m}$ )

$$
h_{i c e}=2.4\left(\sum\left|-T_{a}\right|\right)^{0.5} \text {. }
$$

In this formula $\Sigma\left|-T_{a}\right|$ is the sum of negative average daily temperatures of the air. This dependence was applied [13] to evaluate the rate of the ice cover freezing rate and the ice cover thickness as function of its thickness and the atmospheric air temperature. The 
following formula was derived for the rate of the ice cover freezing (the increasing of ice thickness from initial value $h_{0}$ )

$$
W_{f r}\left(h_{0}, T_{a}\right)=1.66 \cdot 10^{-4}\left(2.73 h_{0}^{-0.68}-1\right)\left|T_{a}\right| .
$$

For calculation of the ice cover thickness $h$ that will be possible to observe through a certain comparatively short time span: $N$ days (the short-term weather forecast), following formula was derived

$$
h=1.66 \cdot 10^{-4}\left(2.73 h_{0}^{-0.68}-1\right) \sum_{i=1}^{N}\left|T_{a i}\right|
$$

All numerical coefficients here are dimension ones to provide the dimension of $W_{f i}-m / d a y, h_{0}$ and $h-m$, accordingly, for results of calculations.

The expression (5) for the ice cover freezing rate gives the possible to evaluate the specific heat flow from the ice crystallization by following formula

$$
Q_{f r}=W_{f r} L_{i c e} \gamma_{i c e} .
$$

In this formula, $L_{i c e}$ - the specific heat of the fresh water ice crystallization, $\gamma_{i c e}$-the density of fresh water ice. This heat flow distributes between the ice cover and water body in accordance with formula (3). After substitution forms (3) and (5) in form (7) following formula for the specific heat flow into the ice cover was derived (dimension is $\mathrm{W} / \mathrm{m}^{2} \cdot{ }^{\circ} \mathrm{C}$ )

$$
Q_{f r}\left(h_{0}, T_{a}\right)=1.66 \cdot 10^{-4} \frac{\gamma_{i c e} \lambda_{i c e} L_{i c e}}{\lambda_{w}+\lambda_{i c e}}\left(2.73 h_{0}^{-0.68}-1\right)\left|T_{a}\right| .
$$

\section{Correlation of Temperature on Air-Snow and Snow-Ice Interfaces with Atmospheric Air Temperature}

Re-arrange formula (1) to define the heat flow only by the difference of the temperature of atmospheric air and water on the border ice-water in following form

$$
q_{w a}=\frac{T_{w}-\left(1+R_{\text {sum }} \mu F h_{0}\right) T_{a}}{R_{\text {sum }}} .
$$

Introduce in the analyze the concept the reduced temperature of the atmospheric air $T_{a h}$, which is connected with real temperature of the air $T_{a}$ by following relation

$$
T_{a h}=\left(1+R_{\text {sum }} \mu F h_{o}\right) T_{a} .
$$

In this case, the problem of the specific flow of heat from the water body to the atmosphere can be transformed in the known problem of the heat transfer through the multilayer plate [11]

$$
q_{w a}=\frac{T_{w}-T_{a h}}{R_{\text {sum }}} .
$$

According to the known solution of this problem [11], the temperature of the snow surface differs from the air temperature and can be determined using the following formula

$$
T_{s n}=T_{a}+\frac{q_{w a}}{\alpha_{s a}}
$$

To simplify the subsequent writing of formulas it is reasonable to introduce the coefficients of the "heat flux" (dimension $\mathrm{m}^{2} \cdot \mathrm{C} / \mathrm{W}$ ) that can be defined for each space: atmosphere, snow and ice by following way

$$
\varepsilon_{a}=\frac{1}{\alpha_{s a}}, \quad \varepsilon_{s n}=\frac{h_{s n}}{\lambda_{s n}}, \quad \varepsilon_{i c e}=\frac{h_{i c e}}{\alpha_{i c e}} .
$$

As result, the coefficient of thermal resistance (coefficient of the heat transfer) is possible to present in following form

$$
R_{\text {sum }}=\varepsilon_{a}+\varepsilon_{\text {sn }}+\varepsilon_{\text {ice }} .
$$

The temperature of the snow surface can be defined by following way

$$
T_{s n}=T_{a}+\varepsilon_{a} q_{w a} .
$$

After substitution expressions (9) and (13) into formula (15) and taking into account that temperature of the fresh water on the border with ice is equal always to the temperature of the freezing $T_{w}=0{ }^{\circ} \mathrm{C}$, the following solution for the temperature of the snow layer surface was derived 

Interfaces and the Atmospheric Air Temperature

$$
T_{s n}=\left(\varepsilon_{s n}+\varepsilon_{i c e}\right)\left(\frac{1}{\varepsilon_{a}+\varepsilon_{s n}+\varepsilon_{i c e}}+\mu F h_{0}\right) T_{a}
$$

For the temperature on the interface snow-ice it is possible to define from the condition: the constancy of heat flow from water body to the atmosphere. It means the heat flow $q_{w a}$ is same on the interface from snow and from ice part, that is

$$
q_{w a}=\lambda_{i c e} \frac{T_{w}-T_{i c e}}{h_{i c e}}=\lambda_{s n} \frac{T_{i c e}-T_{s n}}{h_{s n}} .
$$

Following formula for the temperature of the ice layer upper surface was derived after solution this equation

$$
T_{i c e}=\varepsilon_{\text {ice }}\left(\frac{1}{\varepsilon_{a}+\varepsilon_{s n}+\varepsilon_{\text {ice }}}+\mu F h_{0}\right) T_{a} .
$$

By applying the expressions (16) and (18), it is possible to derive the correlation of the temperature of the snow layer surface with the temperature of the upper surface of the ice layer that depends on the thicknesses of snow and ice layers but does not depend upon the atmospheric air temperature

$$
\frac{T_{s n}}{T_{i c e}}=1+\frac{\lambda_{i c e}}{\lambda_{s n}} \frac{h_{s n}}{h_{i c e}} .
$$

\section{Comparison of Results with the Field Measurements}

To check the adequacy of the obtained results, it was performed the comparison of the calculation results on the derived formulas base with the data of the remote field measurements of the vertical profile of temperature from water body up to the atmosphere on the Heilongjiang (Amur) River during January in 2014 and 2015 years. The detailed description of the applied instruments and methodology of these measurements was presented in Ref. [14]. The ice cover and snow layer thicknesses were defined by processing of measured profiles of temperature. The jump of the temperature gradient connected with the change of the heat conductivity on contacting surface indicates coordinates of the interfaces air-snow, snow-ice and ice water (Fig. 1) (This approach is similar to one in Ref. [12]).

For comparison of the calculation results with data of the long-term measurements the ice thickness and the air temperature it is required to take into account the continuous increasing of the ice thickness. The air temperature can be measured with various (but equal) intervals during a day. With this purpose it is necessary to work in formula (8) the coefficient $k_{d}$, that defines that condition. If the air temperature is recorded each 6 hours in the course of each day, the $k_{d}$ $=1 / 4$, if only one time a day the $k_{d}=1$. Accordingly, if the measurement took place during $N$ days, the array of data will have size $M=N / k_{d}$. It is required to take into account on each step when the air temperature was measured.

Besides it is necessary to introduce into formula the special coefficient $k_{t}$ in order to agree the results of calculations with the data of field measurements because the hydrological conditions in some specific river can differ from the conditions for which the initial formula (4) corresponds (the Siberian Rivers). As result, the formula (6) was converted in following form

$$
h=h_{0}+1.66 \cdot 10^{-4} k_{t} k_{d} \sum_{i=1}^{M}\left(2.73 h_{i}^{-0.68}-1\right)\left|T_{a i}\right| .
$$

Fig. 2 presents the variation of the air temperature during January 2014 (registration one time per day) and the comparison of the processed ice thickness (points) and calculated according to formula (20) values (solid line). Coefficient $k_{t}=0.7$ had been defined on base comparison of calculations and measured data. It is possible to accept the adjustment of measured and calculated thickness of the ice cover as quite satisfactory one including the values of the ice thickness and the behavior that corresponds to the air temperature variation.

During January 6-10 (at 2015), the registration of the vertical profile of the temperature from water body 


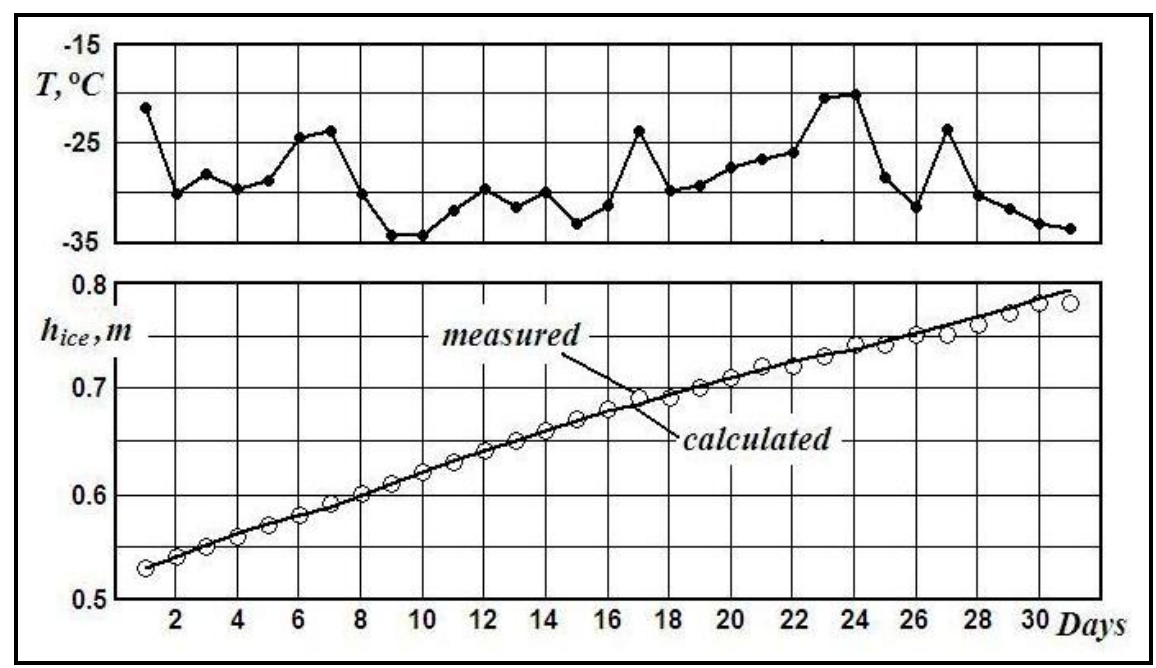

Fig. 2 Comparison of measured (points) and calculated (solid line) thicknesses of ice cover and air temperature (January 2014).

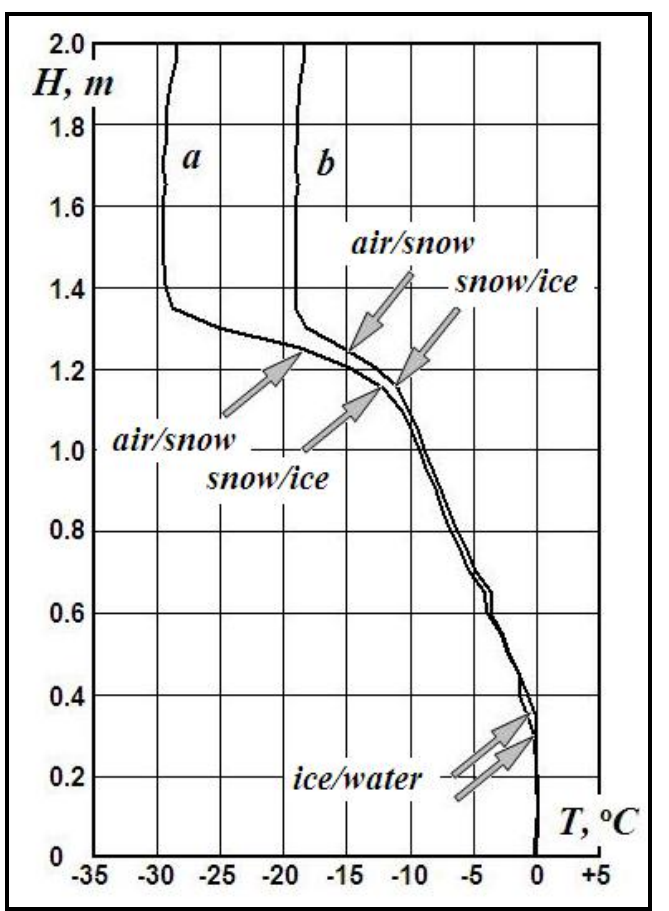

Fig. 3 Vertical profiles of temperature and water-ice, ice-snow and snow-air interfaces (a-16-00 January 10, $b-08-00$ January 6) .

up to the atmosphere and the processing of the ice cover thickness were performed each 8 hours, that was 15 registrations, $k_{d}=1 / 3$. Fig. 3 presents the examples of observed vertical profiles of temperature: $a-16-00$ January 10, 2015 (warming of the weather), and $b$-08-00 January 6, 2015 (became cold). The arrows show the borders of layers, namely, water-ice, ice-snow and snow-atmosphere on the temperature profiles. Vertical axis $(H, m)$ shows the coordinates of sensors along the rod fixed on the river bottom.

Fig. 4 presents the comparison of the ice thickness defined by processing of the measured profiles of temperature (black points) and calculated according formula (20) (solid line) ice thicknesses and the air temperature. Axis $X$ shows the number (№) of the measurement (interval 8 hours) during January 6-10, 2015. Results of the ice thickness calculation correspond

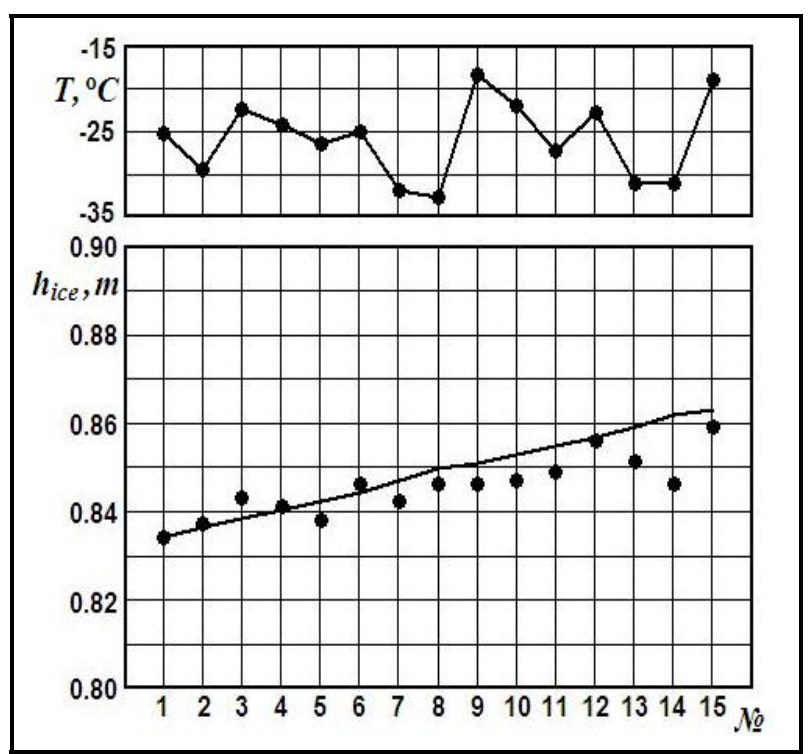

Fig. 4 Comparison of the measured (points) and calculated (solid line) thicknesses of the ice cover and the air temperature (January 6-10, 2015). 
well enough to the ice thickness increasing by the data of the vertical temperature profile processing though the processed data spread is more than on Fig. 2. The thickness of the snow layer during registration time varies within the range of $0.079-0.107 \mathrm{~m}$.

To calculate the temperature of the snow layer surface (16) and the ice cover surface (18) following values of the contained in formulas parameters were accepted [12]:

- Fresh ice heat conductivity $\lambda_{\text {ice }}=2.1 \mathrm{~W} / \mathrm{m} \cdot{ }^{\circ} \mathrm{C}$;

- Snow heat conductivity $\lambda_{s n}=0.44 \mathrm{~W} / \mathrm{m} \cdot{ }^{\circ} \mathrm{C}$;

- Heat-transfer coefficient from snow cover-atmosphere $\alpha_{s a}=6 \mathrm{~W} / \mathrm{m}^{2} \cdot{ }^{\circ} \mathrm{C}$;

- Density of the fresh ice $\gamma_{i c e}=900 \mathrm{~kg} / \mathrm{m}^{3}$;

- Specific heat of phase transition water-ice $L_{i c e}=$ $335 \mathrm{~kJ} / \mathrm{kg}$.

Thicknesses of the snow layer and ice cover were defined from processed data of field measurements.

The most problematic is the choice of the effective heat conductivity of the water space, which defines the value of the heat flow from ice crystallization that passes into the ice cover. Effective heat conductivity differs from the molecular one because it depends upon the rate of turbulent and convective motions in water space. The Heilongiiang (Amur) River is plains river, and according to data [8] the average value $\lambda_{w}=$ $60 \mathrm{~W} / \mathrm{m} \cdot{ }^{\circ} \mathrm{C}$ was accepted. This value provided the satisfactory matching of calculations and data of field measurements.

This parameter defines the part $\mu$ of heat flow in the ice cover owing to freezing ice on the ice-water interface. For accepted $\lambda_{w}$ value $\mu=0.034$. It is small part of the ice crystallization heat but one gives substantial contribution into the total heat flow from the water body to the atmosphere.

Ratio of the snow surface and air temperatures and ratio of the ice surface and air temperatures were calculated applying formulas (18) and (20) and presented (as solid lines) together with measured values (white and black points) in Fig. 5. Top diagram shows the variation of the atmospheric air temperature.

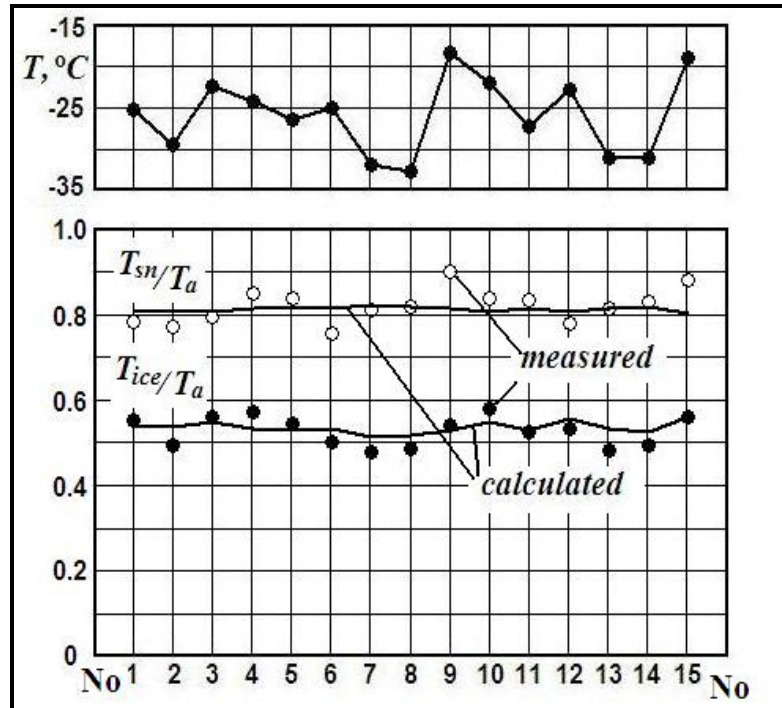

Fig. 5 Measured ratios of snow-air (white points) and ice-air (black points) temperature and calculated ratios (solid line).

After analysis of these results it is possible to draw the conclusion that variation of snow-air and ice-air temperature ratios is substantially smaller than variation of the air temperature during the under consideration time. Average value of the ratio snow-air temperature according to field data is $T_{s n} / T_{a}$ $=0.818$, standard deviation $\sigma=0.04$; according to the results of calculations under formula (16) the ratio $T_{s n} / T_{a}=0.810(\sigma=0.006)$.

Average value of the ratio ice-air temperature according to field data is $T_{i c e} / T_{a}=0.525(\sigma=0.035)$, according the results of calculations under formula (18) ratio $T_{i c e} / T_{a}=0.534(\sigma=0.013)$. The measured data and the results of calculation are sufficiently close.

\section{Analysis of the Results of Modeling}

It is necessary to notice that according to data [2] the ratio of the snow-air temperature is $T_{s n} / T_{a}=0.88$ under maximal thickness of the ice cover on the $\mathrm{Ob}$ and Irtysh Rivers. The dependence of the ratio snow-air temperatures on the relative snow cover thickness $\delta h_{i s}=h_{s n} / h_{i c e}$ and the ice cover thickness $h_{i c e}$ obtained by modeling is presented on Fig. 6 . These data demonstrate that under the ice cover and snow layer thicknesses increasing (that is increasing of the 


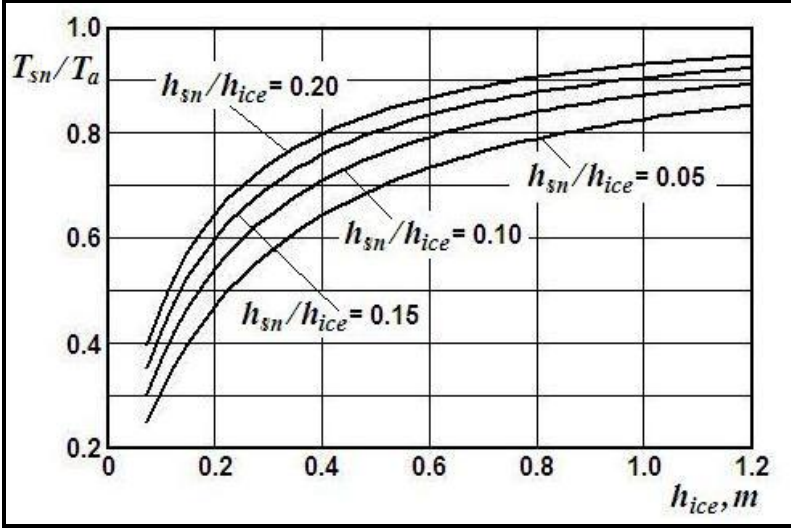

Fig. 6 Dependence of the ratio of the snow surface and air temperatures on the ice thickness for various relative thickness of the snow layer.

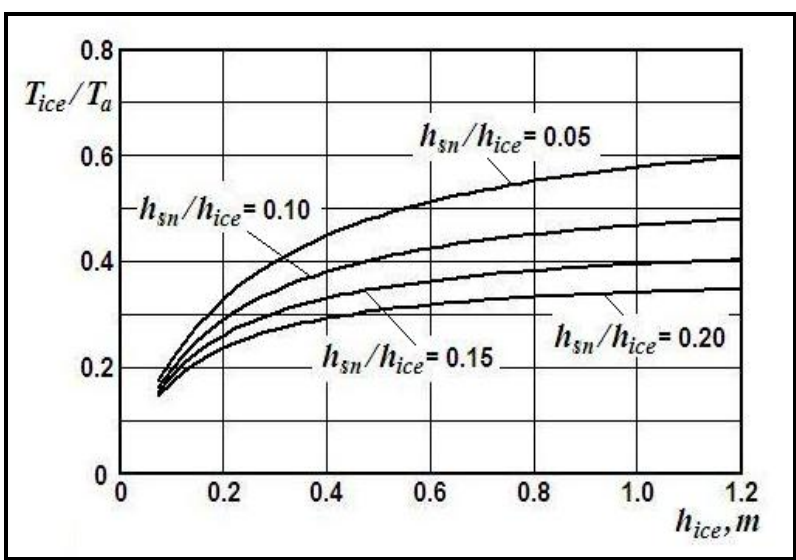

Fig. 7 Dependence of the ratio of the ice cover surface and air temperatures on the ice thickness for various relative thickness of the snow layer.

total insulation layer thickness between water body and atmosphere) the temperature of the surface of the snow layer draws nearer to the air temperature. Therefore presented above and obtained by the way of measurements and calculations the ratio of snow-air temperatures it is possible to consider as consistent data [5].

Fig. 7 presents the results of the ratio ice-air temperatures $T_{i c e} / T_{a}$ modeling in dependence on the thickness of ice cover for various relative snow layer thickness $h_{s n} / h_{i c e}$. These data show that under the snow layer thickness increasing (results of snow falls) the heat-insulating capability of the snow layer growths also, the coefficient of the thermal resistance $R_{\text {sum }}$ increases under formula (2), and as result the difference between the temperature of the ice cover

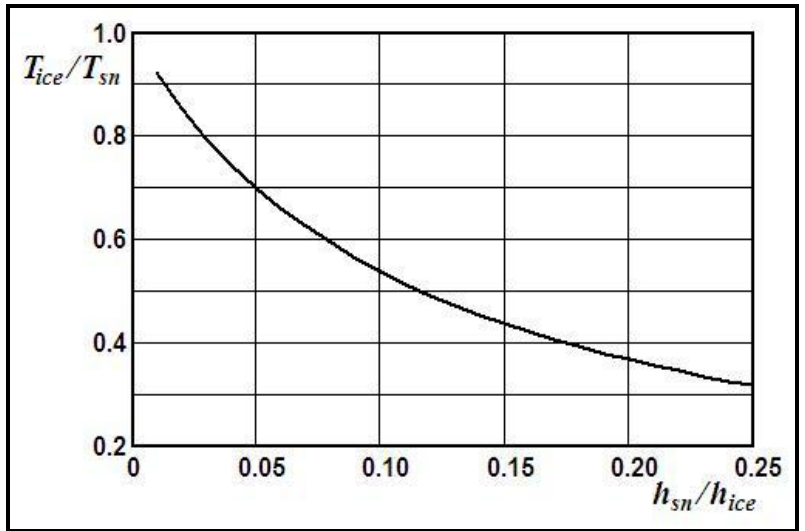

Fig. 8 Dependence of the ratio of the ice cover surface and snow surface temperature on various relative thickness of the snow layer.

surface and the air temperature decreases (the known effect of the thermo-insulation). By turn, this effect slows down the process of ice cover freezing that corresponds to data of the field observations $[3,5]$.

Fig. 8 illustrates this thermo-insulation effect of the snow cover, which is the dependence of the ratio ice-snow temperatures $T_{i c e} / T_{s n}$ on the interface snow layer-ice cover on the relative thickness of the snow layer $h_{\text {sn }} / h_{\text {ice }}$.

\section{Conclusion}

As result of application of the known in the thermophysics solution about the heat conduction through the multilayer plate, it was derived the comparatively simple equations, which relate the temperature of the atmospheric air with the temperature of the snow layer surface and the ice temperature on the snow-ice interface on the fresh water basins.

It was derived the empirical formula which gives possibility to predict the fresh ice cover thickness on the base of the average daily air temperature forecast and the ice cover thickness at the moment.

For a solution to these problems the following assumption was taken on: the quasi-stationary vertical distribution of the temperature in water body, in ice cover and in snow layer that can be realized under the relative slow variation of the air temperature and the sun radiation in the steady winter weather. 
The heat flow from the ice crystallization that takes place on the water-ice interface makes the essential contribution in the vertical profile of temperature forming. For estimation of value and account of the contribution this heat flow, it was carried out the parameterization of the rate of ice cover freezing on the base of the known formula that connects the ice cover thickness with accumulated sum of the negative average daily air temperature for the Siberian Rivers. The heat flow from the ice crystallization was divided between the ice cover and the water body in proportion to their heat conductivity.

Data of the field measurements of the vertical profiles of the temperature (through water-ice-snow-atmosphere) and obtained by processing thickness of ice cover on the Heilongjiang (Amur) River performed in January 2014 and January 2015 years were used to validate the results of the analytical investigations. Performed comparison showed the close fit of the results of calculations and the data of field measurements in the ice cover thickness increasing and the air temperature ratio to the temperature of surface snow layer and surface of the ice cover.

Outcomes of this investigation can be recommended for the practical problems of the ice engineering solution, particularly, for the ice strength evaluation on base of the air temperature data.

\section{Acknowledgement}

Reported investigations were partially supported by the Russian Foundation for Basic researches project No. 15-58-53013 ГФЕН_a and the National Natural Science Foundation of China under contracts No. 51279122 and No. 51511130042.

\section{References}

[1] Green, G. M., and Outcalt, S. I. 1985. "A Simulation Model of River Cover Thermodynamics." Cold Regions Science and Technology 10: 251-62.

[2] Lepikhin, A. P. 2008. "For the Ice Cover Calculation on the Fresh Water Basins." Geographicheskiy Vestnik 1: 13.

[3] Pounder, E. R. 1967. The Physics of Ice. M.: Mir, 190.

[4] Chizhov, A. N. 1990. Formation of the Ice Cover and Spatial Distribution Its Thickness. L.: Gidrometeoizdat, 128.

[5] Donchenko, R. V. 1987. The Ice Regime of the USSR Rivers. L.: Gidrometeoizdat, 242.

[6] Goncharov, V. K., Edush, N. Yu., Zueva, E. S., Klementeva, N. Yu., Qin, J. M., Cui, L. Q., Zhang, L., Deng, X., and Cheng, P. 2016. "Forecast of the Freezing Rate of the Fresh Water Ice Cover." International Conference on Naval Architecture and Ocean Engineering, NAOE2016, 2016, 4.

[7] Lepparanta, M. 2015. Freezing of Lakes and the Evolution of Their Ice Cover. Springer-Verlag, 301.

[8] Velikanov, M. A. 1954. Dynamics of Channel Flow. M.: Gostechizdat, 323.

[9] Qin, J. M., Wang, L. J., Li, X. Ch., Aleshin, Igor V., and Goncharov, Vadim K. "Realization of the Auto-Measurement of Ice in Riverway Based on the Difference of the Air, Ice and Water." In Proceedings of the 230th Symposium on Ice. 14-17 June 2010, Lahti, Finland, CD-ROM. Paper 068, 10.

[10] Shmakin, A. B., Turkov, D. V., and Mikhailov, A. Yu. 2009. "Model of Snow Cover with Inclusion of layered Structure and Its Seasonal Evolution." Earth's Cryosphere XIII (4): 69-79.

[11] Kreiht, F., and Black, W. Z. 1980. Basic Heat Transfer. NY: Harper and Row, 512.

[12] Krass, M. S., and Merzlikin, V. G. 1990. Radiation Thermophysics of the Snow and Ice. L.: Gidrometeoizdat, 262.

[13] Hammonds, K., Lieb-Lappen, R., Baker, I., and Wang, X. 2015. "Investigating the Thermophysical Properties of the Ice-Snow Interface under a Controlled Temperature and Gradient. Part I: Experiments and Observations." Cold Regions Science and Technology 120: 157-67.

[14] River Lake Ice Engineering, edited by Ashton, G. D. 2004. Water Resources Publication, LLC, USA, 478. 